\title{
Three Dimensional Accurate Morphology Measurements of Polystyrene Standard Particles on Silicon Substrate by Electron Tomography
}

\author{
Misa Hayashida ${ }^{1}$, Kazuhiro Kumagai ${ }^{2}$, Marek Malac ${ }^{1,3}$ \\ ${ }^{1}$ National Institute for Nanotechnology (NINT), Edmonton, Alberta, Canada \\ ${ }^{2}$ National Institute of Advanced Industrial Science and Technology (AIST), Tsukuba, Ibaraki, Japan \\ ${ }^{3}$ Department of Physics, University of Alberta, Edmonton, Alberta, Canada
}

Polystyrene latex (PSL) nanoprticles (NPs) spheres are frequently used as size calibration standard over the $30 \mathrm{~nm}$ to $250 \mathrm{~nm}$ range. Their shape is assumed to be spherical. However, atomic force microscopy (AFM) results indicate difference between lateral and vertical dimensions [1]. Lateral dimensions (in the plane of the supporting substrate) are typically larger than the vertical (perpendicular to the substrate plane) dimensions. It is therefore necessary to accurately measure the shape of the PSL nanoparticles. Electron tomography in a (scanning) transmission electron microscope (S)TEM allows to accurately measure three dimensional (3D) shape of nanoscale objects.

A water suspension of PSL nanoparticles (SC-0100-D, JSR Life Science) was deposited onto a silicon substrate, and allowed to dry overnight. To enhance the contrast of NPs in TEM image, they were coated with 20 nm-thick osmium film by plasma-enhanced chemical vapor deposition, followed by 200 nm thick sputtered carbon film. Prior to cutting process by focused ion beam (FIB), the area around target NPs was covered with additional thick carbon layer $(\sim 1.5 \mu \mathrm{m})$ to prevent damage in the cutting process.

A rod-shaped sample with a single PSL NP was fabricated in the FIB to allow data collection without a missing-wedge eliminating the distortions arising from the missing wedge. Nano dot fiducial markers were deposited for high accuracy alignment using electron beam induced deposition of tungsten [2].

A Hitachi HF-3300 TEM/STEM equipped with cold field emission gun was used to collect tomographic tilt series in annular dark field mode (ADF). For the data acquisition, a Matlab ${ }^{\mathrm{TM}}$ based computer control system (MAESTRO) designed for Hitachi electron microscopes was used to control the data acquisition process [3]. At each tilt $512 \times 1024$ pix images with $5 \mu$ s /pixel dwell time and about about 7 pA probe current. The tilt step was $3^{\circ}$ over the entire $\pm 90^{\circ}$ tilt range resulting in 61 images. Four images were acquired at each tilt for a total of $20 \mu$ s per pixel dwell time to decrease effect of sample drift while keeping acceptable signal to noise ratio. The images at each tilt were summed after compensating for drift using cross correlation alignment. Filtered back projection was used to reconstruct the three dimensional volume representation of the nanoparticle.

Figure 1a shows a projected STEM image of a nominal $100 \mathrm{~nm}$ diameter PSL NP embedded in the rodshaped specimen. The bright contrast of the osmium layer, which accurately traces the surface of PSL NP enables us to accurately evaluate the shape the nanoparticle. Figures 1b) to d) show cross-sectional images along three perpendicular planes extracted from the reconstructed volume.

The NP cross sectional shape can be fitted to a circle in a plane perpendicular to the sample rod Y axis. Cross sectional shape in planes parallel to the sample rod $Y$ axis can be fitted to an ellipse. The shape is conveniently obtained by evaluating diameter of a circle fitted to sections perpendicular to $\mathrm{Y}$ axis at known distances from the silicon substrate. The method yields a $93.9 \mathrm{~nm}$ diameter and particle reaches 
$85.5 \mathrm{~nm}$ above the substrate in $\mathrm{Y}$ direction. Therefore the particle is squished by $9 \%$ in the $\mathrm{Y}$ direction becoming an ellipsoid with the short axis in the $\mathrm{Y}$ direction. The measured squish ratio agrees with the AFM measurements of nanoparticle assemblies [1]. Unlike the AFM measurements where the particle shape is inferred from periodicity of nanoparticle assembly on a substrate, here we report direct quantitative measurement of individual nanoparticle shape. The sub $1 \mathrm{~nm}$ resolution obtained here was afforded by the high accuracy alignment [2], high contrast osmium coating and low irradiation dose used for the data collection. The entire process that leads to the nanoparticle dimension measurement can be quantified in terms of accuracy of the individual steps in a traceable manner.

[1] I. Misumi, et. al., SPIE Proceedings, Vol. 8378 (2012), 83780J.

[2] M. Hayashida, M. Malac, M. Bergen' P. Li, Ultramicroscopy, Vol. 144, (2014), 50-57.

[3] M. Bergen et. al. Microscopy and Microanalysis 19 (S2) (2013), 1394-1395.

Ms. M. Ito and Dr. H. Sakurai (AIST, Japan) performed nanoparticle sample preparation. Financial support of Visiting Researcher grant from Alberta Innovates Technology Futures is gratefully acknowledged. This work was done when the first author worked at her earlier affiliation, AIST.

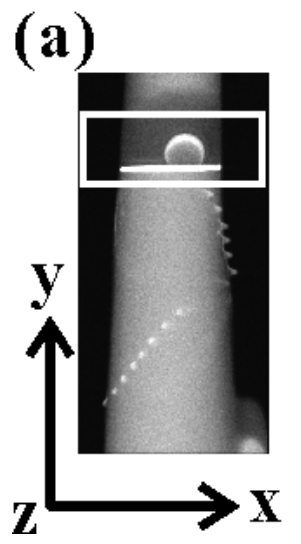

(b)

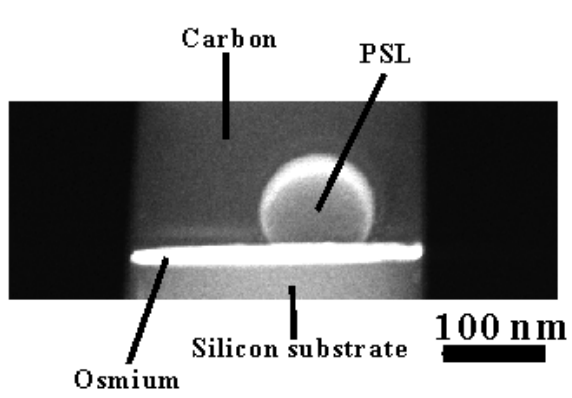

(c)

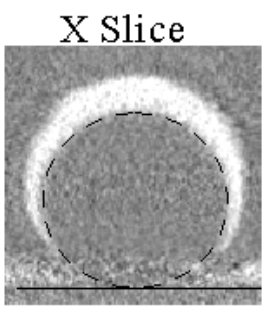

Z Slice

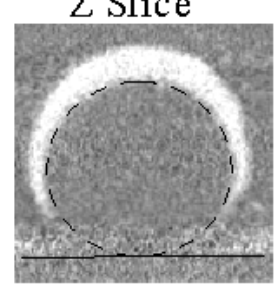

(d)

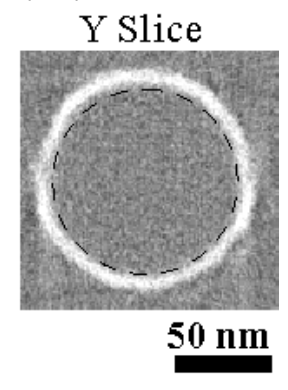

Figure 1. A three dimensional 3D observation of PSL NP with $100 \mathrm{~nm}$ diameter which is included in rod shaped specimen. (a) A selected projection ADF STEM image from the tilt series. The sample consists of (from top) amorphous carbon layer, osmium (bright) on PSL NP. The fiducial nano dot markers are at the lower portion of the left most panel in a). The bright horizontal line between the NP and the silicon substrate is the osmium layer. The coordinate axes are indicated by arrows. The siliconnanoparticle interface is in the y plane perpendicular to the $\mathrm{Y}$ axis, which is parallel to the long axis of the sample. (b), (c) and (d) are example X-plane, Z-plane and Y-plane slices through the reconstructed volume. The dashed curve in (b) to (d) indicates the nanoparticle-osmium boundary that was fitted by an ellipsoid to obtain the nanoparticle 3D shape. The solid line indicates the position of the silicon substrate. 